\title{
BOUNDARY REGULARITY AND EMBEDDED SOLUTIONS FOR THE ORIENTED PLATEAU PROBLEM
}

\author{
BY ROBERT HARDT ${ }^{1}$ AND LEON SIMON
}

Any fixed $C^{2}$ Jordan curve $\Gamma$ in $\mathbf{R}^{\mathbf{3}}$ is known to span an orientable minimal surface in several different senses. In the work of Douglas, Rado and Courant (see e.g. [3, IV, §4]) the minimal surface occurs as an area-minimizing mapping from a fixed orientable surface of finite genus and may possibly have self-intersections. In the work of Federer and Fleming (see e.g. [4, §5]) the minimal surface, which occurs as the support of an area-minimizing rectifiable current, is necessarily embedded (away from $\Gamma$ ) but was not previously known even to have finite genus. Our work in [7], which establishes complete boundary regularity for the latter surface, thus implies that there exists an orientable embedded minimal surface with boundary $\Gamma$. In fact:

THEOREM 1. For any compact orientable $n-1$ dimensional $C^{2}$ embedded submanifold $N$ of $\mathbf{R}^{n+1}$, there exists an orientable bounded stable minimal embedded $C^{1, \alpha}$ (for all $0<\alpha<1$ ) hypersurface $M$ with boundary $N$ so that the closure of $M$ in $\mathbf{R}^{n+1}$ equals $M \cup S$ for some compact set $S \subset \mathbf{R}^{n+1} \sim N$ of Hausdorff dimension $\leqslant . n-7$.

Using the existence theory for area minimizing rectifiable currents $[4,5.1]$ and their interior regularity theory [5, Theorem 1], Theorem 1 follows from our boundary regularity result $[7,11.1]$ :

THEOREM 2. If $U$ is an open subset of $\mathbf{R}^{n+1}, T$ is an $n$ dimensional absolutely area minimizing locally rectifiable current in $U$, and $\partial T$ is an oriented embedded $C^{2}$ submanifold of $U$, then, for some open neighborhood $V$ of spt $\partial T$ in $U, V \cap \operatorname{spt} T$ is an embedded $C^{1, \alpha}$ hypersurface with boundary for all $0<\alpha$ $<1$.

W. K. Allard [1, §5] has proven such regularity near points on the boundary of the convex hull of spt $T$. Boundary regularity in $n=2$ for the unoriented problem [4, 5.3.21] (and so the existence of possibly nonorientable embedded minimal surfaces with boundary) also follows form his work. For $k \geqslant 2, C^{k, \alpha}$

\footnotetext{
Received by the editors June $21,1978$.

AMS (MOS) subject classifications (1970). Primary 49F22, 49F10; Secondary 49F20, $53 \mathrm{~A} 10$.

Key words and phrases. Minimal surface, absolutely area minimizing rectifiable current, tangent cone, excess.

1 Partially supported by NSF Grant MCS-7701747 
smoothness (analyticity) in Theorems 1,2 for $C^{k, \alpha}$ (analytic) boundaries follows from $[8,1.10]$. In proving Theorem 2 , we obtain:

TheOREM 3. Any compact orientable $n-1$ dimensional embedded minimal submanifold of $\mathbf{S}^{n}=\mathbf{R}^{n+1} \cap\{x:|x|=1\}$ with boundary $\mathbf{S}^{n} \cap\left\{\left(x_{1}, \ldots\right.\right.$, $\left.\left.x_{n+1}\right): x_{n}=0=x_{n+1}\right\}$ must be a great hemisphere.

[2, Theorem A] shows that spt $T$ above may have an $n-7$ dimensional interior singular set and that the analogue of Theorem 3 for submanifolds without boundary is false. For $n=2$, Theorem 2 implies:

THEOREM 4. For any $C^{2}$ Jordan curve $\Gamma$ in $\mathbf{R}^{3}$, there exists a nonnegative integer $G_{\mathrm{\Gamma}}$ so that:

(1) The Douglas-Courant type, genus g least-area problem [3, IV, 4.1, 4.4] for $\Gamma$ has no solution whenever $g>G_{\Gamma}$.

(2) There exists a Douglas-Courant type genus $G_{\Gamma}$ least-area solution for $\Gamma$, and any such solution is embedded.

(3) The number of such solutions is finite if $\Gamma$ is $C^{4, \alpha}$.

There are also a priori bounds on $G_{\Gamma}$, the number of solutions, and the absolute value of the Gaussian curvature of any solution.

Sketch of PRoOF of TheOREM 2. To obtain regularity near a point $a \in$ spt $\partial T$, we assume $a=0$ and first prove that the support of some oriented tangent cone at 0 is contained in a hyperplane. For $n=2$, this follows from the monotonicity formula $[1,3.4]$, interior regularity [5], and the planar nature of geodesics on $\mathbf{S}^{2}$. For $n>2$, an inductive argument using linear barriers is required. Letting $H_{ \pm}=\mathbf{R}^{n} \cap\left\{\left(y_{1}, \ldots, y_{n}\right): \pm y_{n}>0\right\}$ and rotating, we assume that for some positive integer $m$ the oriented tangent cone is the sum of $m$ times $H_{+} \times\{0\}$ and $m-1$ times $H_{-} \times\{0\}$, both taken with the usual orientation $\mathbf{e}_{1} \wedge \cdots \wedge \mathbf{e}_{m}$. Since the case $m=1$ has been treated by Allard [1, §5], we henceforth assume $m \geqslant 2$.

Using $[4,5.4 .2]$, we now see that the normalized height

$$
h(r)=\sup \left\{\left|x_{n+1}\right| / r:\left(x_{1}, \ldots, x_{n+1}\right) \in \operatorname{spt} T,\left|\left(x_{1}, \ldots, x_{n}\right)\right| \leqslant r\right\}
$$

has lower limit 0 as $r \downarrow 0$. After establishing that $h(r)$ is comparable (except for a boundary curvature term and a slight change in $r$ ) with the cylindrical excess $\operatorname{Exc}(T, 0, r)$ of $[4,5.3]$, we may apply the interior regularity theorem $[4,5.3 .14]$ in vertical circular cylinders which do not meet spt $\partial T$. From this, one finds $C^{1}$ domains $\Omega_{ \pm} \subset H_{ \pm}$which are mutually tangent at the origin so that over $\Omega_{+} U$ $\Omega_{-}$, spt $T$ separates into graphs of real analytic minimal-surface-equation solutions:

(1) $u_{1}^{+} \leqslant u_{2}^{+} \leqslant \cdots \leqslant u_{m}^{+}$on $\Omega_{+}, \quad u_{1}^{-} \leqslant u_{2}^{-} \leqslant \cdots \leqslant u_{m-1}^{-}$on $\Omega_{-}$. 
Concerning the boundary behavior of each $u_{i}^{ \pm}$, one may, at this stage, only conclude that

$$
\lim _{\Omega_{ \pm} \ni y \rightarrow 0}\left|u_{i}^{ \pm}(y)\right|+\left|D u_{i}^{ \pm}(y)\right|=0 \text {. }
$$

The goal of the middle third of [7] is the specific estimate

$$
\lim _{r \downarrow 0} \sup ^{-1 / 2} h(r)<\infty
$$

Besides involving many well-known concepts of geometric measure theory (monotonicity, excess, blowing-up) and well-known nonparametric regularity estimates (DeGiorgi-Nash, Schauder), the work here includes a new estimate on the radial derivative of each $u_{i}^{ \pm}$and a new comparison between spherical and cylindrical excess.

Using (3), we verify that $\Omega_{ \pm}, u_{i}^{ \pm}$may be chosen so that

$$
\Omega_{ \pm} \text {is a } C^{1,1 / 10} \text { domain, } \quad u_{i}^{ \pm} \in C^{1,1 / 4}\left(C l o s \Omega_{ \pm}\right) \text {. }
$$

Under conditions (1), (2), and (4), the $C^{1, \alpha}$ Hopf-type boundary point lemma of Finn and Gilbarg [6, Lemma 7] implies that $u_{1}^{+}=\cdots=u_{m}^{+}, u_{1}^{-}=\cdots=$ $u_{m-1}^{-}$. For a small open ball $B$ about 0 , we then subtract off the oriented com. ponent, which meets the graph of $u_{1}^{+}$, of the regular points of $B \cap($ spt $T) \sim$ spt $\partial T$ to obtain an area minimizing $S \in R_{n}^{\text {loc }}(B)$ with $\partial S=0$ and spt $S=B \cap$ spt $T$. The proof is completed by using the interior regularity theorem [4, 5.3.18] which implies that (since $h(r) \rightarrow 0$ as $r \downarrow 0$ ) spt $S$ is, near 0 , an embedded real analytic minimal submanifold.

\section{REFERENCES}

1. W. K. Allard, On the first variation of a varifold: boundary behavior, Ann. of Math. (2) 101 (1975), 418-446.

2. E. Bombieri, E. DeGiorgi, and E. Giusti, Minimal cones and the Bernstein prob. lem, Invent. Math. 7 (1969), 243-268.

3. R. Courant, Dirichlet's principle, conformal mapping, and minimal surfaces, Intersciences, New York, 1950. 1969.

4. H. Federer, Geometric measure theory, Springer-Verlag, Berlin and New York,

5. - The singular sets of area minimizing rectifiable currents with codimension one and of area minimizing flat chains modulo two with arbitrary codimension, Bull. Amer. Math. Soc. 76 (1970), 767-771.

6. R. Finn and D. Gilbarg, Asymptotic behavior and uniqueness of plane subsonic flows, Comm. Pure Appl. Math. 10 (1957), 23-63.

7. R. Hardt and L. Simon, Boundary regularity and embedded solutions for the oriented Plateau problem, Ann. of Math. (to appear).

8. C. B. Morrey, Jr., Multiple integrals in the calculus of variations, Springer-Verlag, Berlin and New York, 1969.

SCHOOL OF MATHEMATICS, UNIVERSITY OF MINNESOTA, MINNEAPOLIS, MINNESOTA 55455

MATHEMATICS DEPARTMENT, UNIVERSITY OF MELBOURNE, PARKVILLE, VICTORIA, 3052, AUSTRALIA 